\title{
Facilitating model-building of electrostatics concepts related to conductors
}

\author{
Ryan L. C. Hazelton, Peter S. Shaffer, and Paula R. L. Heron \\ Department of Physics, University of Washington, Box 351560, Seattle, WA, 98195
}

\begin{abstract}
We have been conducting a long-term investigation at the University of Washington into student difficulties with electrostatics concepts, involving students at both the introductory and upper-division level. An important finding is that standard instruction through lecture and textbook does not provide students with a coherent conceptual model that is sufficient to understand the behavior of conductors. We have identified specific difficulties and developed worksheets for both Tutorials in Introductory Physics and Tutorials in Physics on the electric properties of conductors. This paper demonstrates that a teaching strategy designed to help students construct a model for conductors can significantly improve student understanding, at both the introductory and junior levels.
\end{abstract}

PACS: 01.40.Fk, 01.40.Gb

\section{INTRODUCTION}

The physics education community has produced a large body of research on student understanding of circuits and about the development of curriculum and instructional strategies to aid student learning. Research has also been done on early electrostatics concepts (e.g., electric fields and Gauss's law). However, relatively little has been published that relates to intervening topics such as electric potential difference and conductors.

Published findings about conductors indicate that many students have serious problems when reasoning about charges and fields in conductors. Bilak and Singh ${ }^{1}$, for example, found that three-quarters of introductory students and two-thirds of graduate students incorrectly thought that the electric field inside a Faraday cage is non-zero. Guruswamy et $\mathrm{al}^{2}$ found that fewer than a third of students ranging from eighth grade to senior undergraduate courses on E\&M correctly predicted the transfer of charge between two conductors. Students often ignored the forces on a charge in a conductor due to other charges in or on the same conductor. Guisasola et $\mathrm{al}^{3}$ found that students tended to reason about processes involving conductors in terms of charge, not potential differences, in ways that often led them to incorrect answers. For example, three-quarters of introductory students predicted that the equilibrium state of two conductors in contact corresponded to equal amounts of charge on each conductor, as opposed to equal potentials.

Several studies ${ }^{4-6}$ have shown that teaching electric circuits through the use of microscopic models can increase student understanding. Thacker et al, for example, found that in a completely traditional course, simply changing the textbook to one that emphasized microscopic models had a significant effect on student performance. They demonstrated that they could apply the models even to unfamiliar situations. This finding is supported by the work of Christian and Talanquer ${ }^{7}$, who found that chemistry students who used model-based reasoning tended to operate at higher levels of cognitive processing than students who primarily used rule-based or case-based reasoning. The intervention described by Thacker et al explicitly exposed students to model-based reasoning, and most students spontaneously used the model in post-instruction interviews.

The success of model-based instruction in teaching circuits suggests that similar instructional strategies may be effective in helping students learn to reason about electric charge, field, and potential difference in conductors. We have developed curriculum to try to help students construct models they can use to predict the electric behavior of conductors. The results from pretests and post-tests indicate that the majority of students switched from using rule-based reasoning before tutorial to using model-based reasoning after working through the tutorial.

\section{CONTEXT FOR RESEARCH}

This study was conducted at the University of Washington in the large calculus-based introductory physics course and in the upper-division E\&M course. The latter course used Griffiths' Introduction to Electrodynamics. Both courses had 3 hours of lecture each week, and weekly 50-minute tutorial sections. The tutorial sections had between 18 and 26 students, who worked in groups of 3-5 on worksheets from either Tutorials in Introductory Physics ${ }^{8}$ or Tutorials in Physics: Electrodynamics ${ }^{9}$. Two graduate teaching assistants taught each tutorial. The introductory course also had standard weekly 2-hour laboratory sections.

In both courses, students took weekly on-line "pretests" (given after lecture instruction) to gauge their initial understanding of the topics to be covered in the upcoming tutorial. After each tutorial, students completed tutorial homework designed to strengthen their understanding of the ideas covered. The midterm and final exams each included questions based on the tutorials, and in both classes a midterm was given two weeks after the relevant tutorial. 


\section{DESCRIPTION OF CURRICULUM}

We have developed a tutorial worksheet for Tutorials in Introductory Physics, called Electric properties of conductors. Topics covered include: the net electric field, induced electric fields, and potential differences in conductors. The tutorial also guides students in applying these ideas to grounding. This worksheet was developed through several iterative rounds of research into student ideas about conductors. Each round was validated by comparing pretest and post-test data, to test the net effect after each addition or modification.

In the tutorial, students are given the equipment shown in Fig. 1, which consists of a polarizable "pith ball" made of plastic foam, a plastic rod that students charge by rubbing with wool, and a foam cup that is lined with aluminum foil. Students begin by bringing the neutral pith ball near the rod and observe its behavior. The pith ball polarizes and is attracted toward the rod. Students then repeat the experiment with the pith ball suspended inside the cup and find that the pith ball experiences no net force. The rest of the tutorial guides the development of a model to explain these observations.

Students are reminded of the superposition principle, and asked to use their observations to determine the net electric field inside the cup. They are then reminded that charges are free to move in a conductor and are asked to discuss how the conductor reacts to an external charge, and to find the induced charge distribution on the cup. The emphasis throughout the tutorial is that the charges in a conductor react to an external field, to create an induced field opposite that of the external field. This mechanistic model of charge movement is then used to explain more complicated ideas such as grounding and charging by induction.

An analogous tutorial was developed for Tutorials in Physics: Electrodynamics. There are two key differences: thought experiments are used instead of physical equipment, and the development of the concept of shielding replaces the section on grounding.

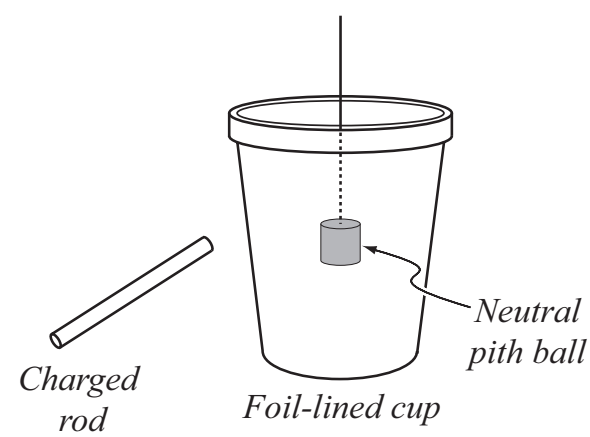

FIG 1. Equipment used for the electric field experiments in the tutorial Electric properties of conductors.

\section{DATA AND DISCUSSION}

Throughout this paper, data from multiple classes given during different quarters are combined since the distributions of answers are almost identical. Chi-squared analyses show no statistical differences between any of the classes that were combined. Not every class had similar versions of any given question, so the number of responses to each question varies. More than $85 \%$ of students answered each question; the populations that answered each question were also not statistically significant.

\section{A. Results from the introductory class}

\section{Before tutorial: Field inside conductors}

Prior to working through the tutorial, students in the introductory course took a pretest, in which two questions were based on the scenario shown in Fig. 2. The first question asked students to predict the direction of the net electric field at point $P$. The second question asked about the sign of the potential difference from the left end of the rod to the right end of the rod (i.e., $V_{\text {Right }}-V_{\text {Left }}$ ). Students were asked to explain their answers to both questions.

Of the 640 students who took the pretest, only $47 \%$ correctly stated on the first question that the net field is zero at point $P$. About one-third stated that the net field is to the left due to the point charge. On the second question, 35\% of the students correctly answered that the potential difference was zero. However, only $25 \%$ of the students overall had correct answers and correct reasoning for both questions.

Nearly half the students gave inconsistent answers in their choice for the direction of the field and in their explanation. For instance, $16 \%$ indicated that the net field is to the right but then stated that the field is zero in their explanation. These students often explained with very brief statements such as "E is zero."

Twenty-one percent of the students stated that the electric field at point $P$ is zero, but then stated that the potential difference across the rod is non-zero. These students did not use their correct answer about the field, but instead used the formula for a point charge, $\mathrm{kQ} / \mathrm{r}$.

Both groups of students above can be categorized as using rule-based thinking instead of model-based thinking. They are stating a rule $(\mathrm{E}=0$ or $\mathrm{V}=\mathrm{kQ} / \mathrm{r})$ and then either misapplying the rule or forgetting the circumstances that the rule describes. Only a quarter of the students seemed to be correctly using model-based reasoning for both questions, i.e. using their knowledge about a conductor (the net field is zero or charges in equilibrium don't move) as the base for a chain of reasoning to answer the question.

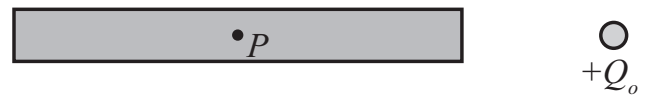

FIG 2. A positive charge at rest next to a conducting rod. 


\section{Before tutorial: Grounding}

A question that probed ideas similar to those on the pretest was asked in a different class on a post-laboratory assignment, given after students had completed all relevant lecture and lab instruction. The setup for the post-lab question is almost identical to that for the question described in Fig. 2, except the conducting rod is grounded. The question asked about the net charge on the conducting rod.

Of the 305 students who took the post-lab, $60 \%$ correctly answered that the conductor was net negative, and $35 \%$ incorrectly stated that the conductor was neutral. Most of the latter students explicitly stated in their explanations that ground neutralizes objects, despite the fact that this is in contradiction to what they had seen in the lab. These students seem to answer on the basis of a memorized response that "ground neutralizes," independent of the arrangement and connections within a charged system and can be characterized as using rule-based thinking.

\section{After tutorial: Field inside conductors}

We have administered a variety of post-test questions after students worked through the tutorial. The post-test shown in Fig. 3 is one example, which has been given to 234 students. On this post-test, a negative point charge is quickly placed near a neutral metal sphere attached by a conducting wire to ground. Students were asked about the direction of the net field in the sphere and wire, (a) before and (b) after equilibrium. The correct answer is that before equilibrium the fields are non-zero since the charges in the conductors have not yet moved, and are zero at equilibrium.

Sixty-five percent of the students correctly answered both questions using language consistent with model-based reasoning. This compares favorably with student performance on the pretest, for which about half of the students gave the correct answer and only a quarter had correct reasoning. This fraction is similar to results on the other post-test questions.

\section{After tutorial: Grounding}

On the post-test shown in Fig. 3, students were also asked about the charge on the sphere and the potential difference from the sphere to ground, in equilibrium.

Seventy-two percent of students correctly answered both questions. $40 \%$ gave the correct answer and used language consistent with applying the model used in the tutorial:

"The sphere makes a field to cancel the field of the negative charge, so the induced field points along the wire."

"Connecting the sphere to the earth means they must be equipotentials since the net field is zero inside a conductor, and now the sphere and earth are just one big conductor."

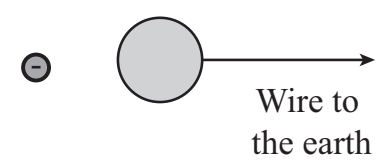

FIG 3. A negative charge is suddenly placed near a grounded conducting sphere.

The proportion of students who stated rules or used reasoning consistent with applying rules was much lower than in the pretest. $13 \%$ of students incorrectly stated that the sphere was neutral, and $18 \%$ incorrectly stated that the potential difference would be nonzero. Overall, only $15 \%$ of students stated rules in their explanation, compared to between $35 \%$ and $45 \%$ on the pretest.

Overall, the introductory students perform significantly better on field and potential questions about conductors after working through the tutorial than before the tutorial. The tutorial also seems to help students switch from simple rule-based thinking to more complex thinking.

\section{B. Results from the junior class}

A variety of questions have been given to students in the junior level course, after students had seen traditional lecture instruction on conductors. Some of these have been used as tutorial pretests, and others have appeared on midterm or final exams after students have worked through the tutorial and associated homework. We have also analyzed student responses on tutorial homework. Below we present results from all three types of assessments.

\section{Before tutorial}

Two of the questions used for tutorial pretests are shown in Fig 4. In the first question (Fig. 4a) students are shown a conducting sphere with a small cavity that contains a positive point charge. They are asked if it is possible to determine the location of the cavity by measuring the electric field outside the sphere. In the second question (Fig. 4b) students are shown a positive charge at several different locations inside a conducting spherical shell, and asked about the charge densities on the inner and outer surfaces of the shell.

On the first pretest question, only about half of the students $(N=165)$ correctly identified that because the sphere in Fig. 4a would have zero electric field throughout it, it would effectively shield the exterior of the sphere from the point charge. Thus, it is not possible to determine the

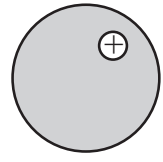

(a)

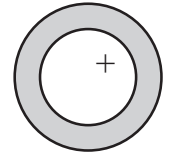

(b)
FIG 4. (a) A charged cavity inside a spherical conductor (b) an off-center charge inside a spherical shell. 
location of the cavity. Most of the students who gave incorrect answers correctly determined that negative charges would accumulate on the inside surface of the cavity. However, when finding the charge distribution on the outside of the sphere, students tended to ignore either the point charge or the charge distribution on the inside of the cavity.

The second pretest question (Fig. 4b) was significantly harder for students than the first. Only $18 \%$ of students correctly stated that the charge distribution would be asymmetrical on the inside surface of the shell and uniformly distributed on the outside surface if the positive charge were off-center. Only when the charge was centered did most students find the correct charge distribution.

\section{Tutorial homework}

One of the questions on the tutorial homework used in the junior level course is based on an experiment similar to one used in the tutorial. (See Fig. 1.) Students are told that a metal ball is suspended inside a metal cylinder, and a charged object is placed outside the cylinder. Out of 81 students, $65 \%$ correctly stated that there would be no induced charge distribution on the ball. These students all correctly reasoned that charges in the cylinder would spontaneously redistribute to cancel the external field, which is precisely the model introduced in the tutorial. This is an improvement over the result of Bilak and Singh, who found that only one-third of first-year graduate students correctly answered a similar question after traditional instruction. Instructional strategies based on model-building seem to be clearly beneficial to students' understanding of this topic.

\section{After tutorial: Post-test on field in a conducting shell}

A variant of the situation shown in Fig. $4 \mathrm{~b}$ was used on a post-test in the junior level course. Students were asked to sketch the charge densities on the inner and outer surfaces of the conducting shell, as well as the electric fields inside and outside. Out of 94 students, 68\% found the correct charge distribution and fields, and 50\% used the logical structure provided by the tutorial to determine their answers. This is a significant improvement from the pretest, on which only $18 \%$ of the students gave the correct answers.

[1] J. Bilak and C. Singh, PERC Proceedings (2007)

[2] C. Guruswamy, M. D. Somers, and R. G. Hussey, Phys. Educ. Vol. 32, p. 91 (1997)

[3] J. Guisasola, J. L. Zubimendi, J. M Almudi, and M. Ceberio, Sci. \& Edu. Vol. 11, p. 247 (2002)

[4] B.-S. Eylon and U. Ganiel, Int. J. Sci. Edu. Vol. 12, p. 79 (1990)

[5] B. A. Thacker, U. Ganiel, and D. Boys, Am. J. Phys. Vol. 67, p. S25 (1999)
In both the introductory and advanced versions of the tutorial, spontaneous use of model-based reasoning after tutorial is significantly enhanced. However since the introductory class has around $5 \%$ of physics majors and the junior class is entirely physics majors, direct comparison across classes is difficult.

\section{CONCLUSIONS AND IMPLICATIONS FOR INSTRUCTION}

The findings from this research indicate that instruction that focuses on explicit model-building to create a causal model of conductors can strengthen the ability of students to reason about charge distributions, electric fields, and potential differences in conductors. It also seems to help students transition from using rule-based reasoning, which is often incorrectly applied, to using model-based reasoning. This strategy can be effective in introductory service courses as well as in courses for upper-division physics majors.

This particular study used a tutorial implementation, but that this technique is similar to that used by Eylon et al suggests that it can be effective in many contexts, not only with classes using tutorial-based instruction. In our view the major significance of this study is that guiding students to construct a model for charges in a conductor leads to more complex reasoning about this topic. This guidance could be implemented via other methods as well, for instance through an appropriate set of clicker questions and discussion, or in a 'flipped' classroom setting.

Having students construct a causal model for circuits has previously been shown to be effective in helping students reason about the behavior of circuits. We think it likely that engaging students in model-building at an earlier stage, starting with conductors, and then continuing the model development with instruction about circuits, could be even more productive than using a causal model only for circuits. We will explore this in future work.

\section{ACKNOWLEDGEMENTS}

The authors would like to acknowledge the Physics Education Group at the University of Washington, and the instructors who graciously allowed this work in their classes, especially Leslie Rosenberg.

[6] P. E. Hirvonen, Eur. J. Phys. Vol. 28, p. 581 (2007)

[7] K. Christian and V. Talanquer, Chem. Educ. Res. Pract. Vol 13, p. 286 (2012)

[8] L. C. McDermott, P. S. Shaffer, and the Physics Education Group at the University of Washington, Tutorials in Introductory Physics. (Prentice Hall, New Jersey, 2002).

[9] L. C. McDermott, P. S. Shaffer, and the Physics Education Group at the University of Washington, Tutorials in Physics: Electrodynamics. (Unpublished) 